\title{
Pyrroloquinoline quinone attenuates isoproterenol hydrochloride-induced cardiac hypertrophy in AC16 cells by inhibiting the NF-kB signaling pathway
}

\author{
JUNRU WEN $^{1,2^{*}}$, JUNWEI SHEN $^{3 *}$, YAJIE ZHOU $^{4}$, XIANHUI ZHAO ${ }^{1,2}$, ZHENSHENG DAI $^{5}$ and YUELING JIN $^{2}$ \\ ${ }^{1}$ Graduate School, Shanghai University of Traditional Chinese Medicine, Shanghai 201203; \\ ${ }^{2}$ Department of Science and Technology, Shanghai University of Medicine and Health Sciences, Shanghai 200237; \\ ${ }^{3}$ Key Laboratory of Arrhythmias, Ministry of Education, Tongji University Affiliated EAST Hospital, Shanghai 200120; \\ ${ }^{4}$ Graduate School, Guizhou Medical University, Guiyang, Guizhou 550025; ${ }^{5}$ Department of Oncology, \\ Shanghai Pudong Hospital Affiliated to Fudan University, Shanghai 200090, P.R. China
}

Received April 23, 2019; Accepted December 11, 2019

DOI: $10.3892 /$ ijmm.2020.4463

\begin{abstract}
Pyrroloquinoline quinone (PQQ) is a naturally occurring redox co-factor that functions as an essential nutrient and antioxidant, and has been reported to exert potent anti-inflammatory effects. However, the therapeutic potential of PQQ for isoproterenol hydrochloride (Iso)-induced cardiac hypertrophy has not yet been explored, at least to the best of our knowledge. In the present study, the anti-inflammatory effects of PQQ were investigated in Iso-treated AC16 cells, a myocardial injury cellular model characterized by an increase in the apparent surface area of the cells and the activation of intracellular cardiac hypertrophy-associated proteins. The results revealed that pre-treatment with PQQ significantly inhibited the expression of cardiac hypertrophy marker proteins, such as atrial natriuretic peptide, brain natriuretic peptide and $\beta$-myosin heavy chain. PQQ also inhibited the activation of the nuclear factor $(\mathrm{NF})-\kappa \mathrm{B}$ signaling pathway in Iso-treated AC16 cells, thus inhibiting the nuclear translocation of $\mathrm{NF}-\kappa \mathrm{B}$ and reducing the phosphorylation levels of p65. On the whole, the findings of this study suggest that PQQ may be a promising therapeutic agent for effectively reversing the progression of cardiac hypertrophy.
\end{abstract}

Correspondence to: Dr Yueling Jin, Department of Science and Technology, Shanghai University of Medicine and Health Sciences, 279 Zhouzhu Road, Pudong, Shanghai 200237, P.R. China

E-mail: jinyl@sumhs.edu.cn

Dr Zhensheng Dai, Department of Oncology, Shanghai Pudong Hospital Affiliated to Fudan University, 2800 Gongwei Road, Pudong, Shanghai 200090, P.R. China

E-mail: zhenshengdai@126.com

*Contributed equally

Key words: pyrroloquinoline quinone, isoproterenol hydrochloride, cardiac hypertrophy, reactive oxygen species

\section{Introduction}

Cardiovascular disease is the disease with the highest associated mortality rate worldwide $(1,2)$. According to data from the World Health Organization, 17.9 million individuals succumb to cardiovascular disease each year, which is estimated to account for $31 \%$ of global mortality. Heart failure is the leading cause of cardiovascular-associated mortality, and myocardial hypertrophy is a common pathological stage in numerous cardiovascular diseases, which is widely recognized as a risk factor leading to cardiac dysfunction or eventual heart failure (3). The increased area of myocardial cells is the major characteristic of cardiac hypertrophy, which is divided into physiological cardiac hypertrophy and pathological hypertrophy. The former is an adaptive response to preserve cardiac function and is initially reversible, while it may develop into irreversible pathological hypertrophy as the disease progresses $(4,5)$. Thus, if it could be effectively reversed to normal conditions without delay before the condition develops to an irreversible one, this may effectively prevent myocardial infarction.

The development of multiple diseases is accompanied by alterations in the levels of biomarkers. For cardiac hypertrophy and heart failure, the biomarkers are atrial natriuretic peptide (ANP), brain natriuretic peptide (BNP) and $\beta$-myosin heavy chain $(\beta-\mathrm{MHC})$, whose levels increase with the progression of cardiac hypertrophy (6). Isoproterenol hydrochloride (Iso) is an agonist of the $\beta$-adrenergic receptor, as well as a model widely used to mimic persistent adrenergic stimulation that can trigger maladaptive cardiac hypertrophy accompanied by the reactivation of ANP, BNP and $\beta$-MHC. These alterations are in accordance with the development of myocardial hypertrophy (7).

Pyrroloquinoline quinone (PQQ) is an anionic water-soluble complex that was originally identified as a methanol dehydrogenase coenzyme (8) and can be isolated from methylotrophic bacteria. PQQ has the functions of protecting neurons, stimulating immunity, and has anti-inflammatory, antioxidant, anticancer and anti-aging properties (9-14), which 
are mediated by a decrease in the release of inflammatory factors and an attenuation of the activation of nuclear (NF)- $\kappa \mathrm{B}$ phosphorylation, thus attenuating inflammatory diseases (12). In addition, PQQ has been shown to exert effective cardioprotective effects, and it has been shown to be more effective in protecting the mitochondria from ischemia/reperfusion oxidative damage than metoprolol, an effective cardioprotective drug (15). A previous study demonstrated that PQQ can modulate the number and function of the mitochondria in mice, and can directly react with reactive oxygen species (ROS) in the mitochondria to maintain the dynamic balance of ROS (16). However, to date, at least to the best of our knowledge, there are only few reports on the effects of PQQ on cardiac hypertrophy (17-21). Based on previous studies (17-21), it was hypothesized that PQQ may be protective against Iso-induced cardiac hypertrophy.

There are numerous studies on the pathogenesis of cardiac hypertrophy focusing on myocardial pressure overload, myocardial apoptosis, vascular remodeling, oxidative stress and the inflammatory response $(4,22-26)$; however, the pathogenesis of cardiac hypertrophy has not yet been fully clarified. The inflammatory response is one of the research hotspots in the pathogenesis of cardiac hypertrophy (26). Previous studies have demonstrated that the activation of the $\mathrm{NF}-\kappa \mathrm{B}$ signaling pathway is closely associated with the inflammatory response, and it has been reported that NF- $\kappa \mathrm{B}$ is involved in vascular remodeling, apoptosis and oxidative stress (27-30). In addition, previous studies have confirmed that Iso can cause cardiac hypertrophy by activating the $N F-\kappa B$ signaling pathway, while the inhibition of $N F-\kappa B$ activation can counteract Iso-induced hypertrophy $(7,31)$. The activation of the $\mathrm{NF}-\kappa \mathrm{B}$ signaling pathway is marked by $N F-\kappa B$ degradation and entry into the nucleus (32). The present study thus aimed to examine the expression of NF- $\kappa \mathrm{B}$ in Iso-induced cardiac hypertrophy following pre-treatment $\mathrm{PQQ}$ in order to confirm the activation of the $\mathrm{NF}-\kappa \mathrm{B}$ signaling pathway and to detect ROS levels in cardiomyocytes. This study also aimed to explore the potential role of PQQ in Iso-induced cardiac hypertrophy.

\section{Materials and methods}

Chemicals, reagents and kits. The human myocardial cell line, AC16 (33), and the rat myocardial cell line, H9c2 (CRL-1446), were obtained from the American Type Culture Collection (ATCC). Dulbecco's modified Eagle's medium (DMEM; C11995500BT) and fetal bovine serum (FBS; 10270-106) were purchased from Gibco (Thermo Fisher Scientific, Inc.) Penicillin-streptomycin solution (PSS) (100X; E607011-0100) was purchased from Sangon Biotech (BBICo., Ltd.). Iso (I5627) and PQQ (D7783) were purchased from Sigma-Aldrich (Merck KGaA). Anti-ANP antibody (GTX109255) was acquired from GeneTex, Inc. Anti-GAPDH antibody (10900R) was obtained from BIOSS. Anti-p65 (10745) antibody, which is an antibody

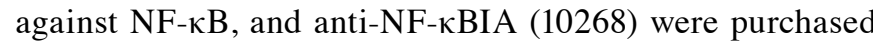
from ProteinTech Group, Inc. Anti-histone-3 (H3; ab1791) antibody was obtained from Abcam. Horseradish-peroxidase (HRP)-conjugated secondary antibody (BA1054) was purchased from Wuhan Boster Biological Technology, Ltd. RIPA lysis buffer (P0013) was purchased from the Beyotime
Institute of Biotechnology. The Cell Counting Kit-8 (CCK-8) kit (40203ES76) was purchased from Yeasen (Yeasen Biotech Co., Ltd.). Reverse transcription-quantitative (RT-q) PCR primers were synthesized by Thermo Fisher Scientific, Inc., while the PrimeScript ${ }^{\mathrm{TM}}$ RT Reagent kit (Perfect Real Time) (RR037Q) and TB Green ${ }^{\mathrm{TM}}$ Advantage ${ }^{\circledR}$ qPCR Premix (639676) were acquired from Takara Bio, Inc. A bicinchoninic acid (BCA) protein quantitation kit (P0010), a reactive oxygen species assay kit (S0033) and the Actin-Tracker Green kit (C1033) were purchased from the Beyotime Institute of Biotechnology. All other chemicals used in the experiments were of analytical grade.

Experimental animals. The animal procedures were approved by the Animal Care and Use Committee of Shanghai University of Medicine and Health Sciences (Shanghai, China; permit no. 210105197306201429-kjc) and were in accordance with the Guide for the Care and Use of Laboratory Animals published by the US National Institutes of Health (NIH) (34). Eighteen C57BL/6 male mice ( 8 weeks old) were obtained from the Animal Resource Center of Shanghai University of Medicine and Health Sciences. All mice were housed in a constant environment with $55 \pm 10 \%$ humidity, a temperature of $20 \pm 5^{\circ} \mathrm{C}$ and a $12 \mathrm{~h}$ light/dark cycle. Adequate food and water were provided ad libitum. The health and behavior of the mice were monitored in real-time.

Animal grouping. In this study, SPF grade C57 male mice (weighing 22-25 g) were used to establish the experimental model (35-38). First, 18 mice were randomly divided into 3 groups (6 mice per group). The treatment groups were as follows: The sham group in which mice received an intraperitoneal injection of normal saline (NS) $100 \mu \mathrm{l} /$ time, and $3 \mathrm{~h}$ later, an intraperitoneal injection of physiological saline $100 \mu \mathrm{l} /$ time; the Iso $(30 \mathrm{mg} / \mathrm{kg})$ group [the doses of Iso were in accordance with those of a previous study (39)] in which mice received an intraperitoneal injection of NS $100 \mu \mathrm{l} /$ time, and $3 \mathrm{~h}$ later, an intraperitoneal injection of ISO dissolved in NS $100 \mu \mathrm{l} /$ time; the PQQ (40 mg/kg) [the doses of PQQ were in accordance with those of a previous study (40)] + Iso (30 mg/kg) group in which PQQ dissolved in NS was intraperitoneally injected into the mice $100 \mu \mathrm{l} /$ time, and $3 \mathrm{~h}$ later, ISO dissolved in NS was intraperitoneally injected into the mice $100 \mu \mathrm{l} /$ time. The above-mentioned managements were performed once every 4 days, and the duration of the experiment was approximately 5 weeks. Following anesthesia by an intraperitoneal injection of $3 \%$ sodium pentobarbital at a dose of $40 \mathrm{mg} / \mathrm{kg}$, the mice were examined to confirm that they were in a state of deep anesthesia; the adequacy of the anesthesia was detected by pinching the hindfoot to ensure there was no reflex, and the heart was then harvested.

Hematoxylin and eosin $(H \& E)$ staining. The recovered heart tissue was rinsed with NS, fixed with $4 \%$ paraformaldehyde for $1 \mathrm{~h}$ at room temperature, and subsequently, $H \& E$ staining was performed at room temperature (hematoxylin, $7 \mathrm{~min}$; eosin, $5 \mathrm{~min}$ ) to observe the pathological changes of myocardial tissue and the images were observed using a digital pathological scanner (Precice 500; UNIC Technologies, Inc.) at $\mathrm{x} 200$ magnification. 
Cell culture and grouping. Either the AC16 cells or H9c2 cells were cultured in a 6-well plate with $2 \mathrm{ml}$ DMEM containing $1 \mathrm{~g} / \mathrm{l}$ glucose, $10 \% \mathrm{FBS}$ and $1 \% \mathrm{PSS}$, and incubated at a constant temperature of $37^{\circ} \mathrm{C}$ in a humid atmosphere of $95 \%$ air and $5 \% \mathrm{CO}_{2}$. Once the cells had adhered to the wall, they were grouped as follows: i) The control group, which were treated with 2 ml DMEM without FBS (DMEMNF, DMEM NO FBS) as a blank control for $48 \mathrm{~h}$; ii) the Iso group, which were treated with $2 \mathrm{ml} \mathrm{DMEMNF}$ for $24 \mathrm{~h}$ and then with $20 \mu \mathrm{M}$ Iso in $2 \mathrm{ml}$ DMEMNF for $24 \mathrm{~h}$; iii) the low-dose PQQ group, which, after being cultured for $21 \mathrm{~h}$ in $2 \mathrm{ml}$ DMEMNF, were treated with $1 \mu \mathrm{M}$ PQQ in $2 \mathrm{ml} \mathrm{DMEMNF}$ for $3 \mathrm{~h}$ and then with $20 \mu \mathrm{M}$ Iso in $2 \mathrm{ml}$ DMEMNF for $24 \mathrm{~h}$; iv) the mid-dose group, which, following $21 \mathrm{~h}$ of culture in $2 \mathrm{ml}$ DMEMNF, were treated with $2.5 \mu \mathrm{M}$ PQQ in $2 \mathrm{ml}$ DMEMNF for $3 \mathrm{~h}$ and then with $20 \mu \mathrm{M}$ Iso in $2 \mathrm{ml}$ DMEMNF for $24 \mathrm{~h}$; and v) the high-dose group, which, after $21 \mathrm{~h}$ of culture in $2 \mathrm{ml}$ DMEMNF, were treated with $5 \mu \mathrm{M}$ PQQ in $2 \mathrm{ml}$ DMEMNF for $3 \mathrm{~h}$ and then with $20 \mu \mathrm{M}$ Iso in $2 \mathrm{ml}$ DMEMNF for $24 \mathrm{~h}$ (Fig. S1).

Cell viability measurements. To explore the cytotoxicity of Iso and PQQ on the AC16 cells, cell viability was determined using a rapid and sensitive CCK-8 assay kit according to the manufacturer's instructions. The AC16 cells were seeded into 96-well plates at a density of $5 \times 10^{3}$ cells per well. After $24 \mathrm{~h}$, Iso was added to the 96-well plates at increasing concentrations $(5,10,20,40,80$ and $160 \mu \mathrm{M})$ and each concentration had 6 set parallel groups, and cultured in an incubator at $37^{\circ} \mathrm{C}$ with a humid atmosphere of $95 \%$ air and $5 \% \mathrm{CO}_{2}$ for $24 \mathrm{~h}$. Moreover, PQQ was added to other 96-well plates at a concentration of $1,5,10,25,50,100,150$ and $200 \mu \mathrm{M}$ setting 6 parallel groups as well, and cultured for $12 \mathrm{~h}$. The medium was then aspirated from the plates, which were rinsed gently with PBS before being filled with CCK- 8 reagent and DMEM at a ratio of 1:10 in $100 \mathrm{ml}$. The plates were incubated for $2 \mathrm{~h}$ in an incubator at $37^{\circ} \mathrm{C}$ and the optical density (OD) value at a wavelength of $450 \mathrm{~nm}$ was measured using a microplate reader (SpectraMax M5; Molecular Devices, LLC).

Cell morphometric analysis. To examine the effect of PQQ against Iso-induced hypertrophy, visual experimentation was carried out. First, either the AC16 cells or $\mathrm{H} 9 \mathrm{c} 2$ cells were plated in 6-well plates, and cultured in a humid atmosphere of $95 \%$ air and $5 \% \mathrm{CO}_{2}$ at a constant temperature of $37^{\circ} \mathrm{C}$. The cells were then treated as described above in the section entitled 'Cell culture and grouping'. The cells were then washed with PBS thrice (5 min each wash) before being fixed in a $4 \%$ paraformaldehyde solution. After $10 \mathrm{~min}$, the $4 \%$ paraformaldehyde solution was aspirated, and $0.1 \%$ Triton X-100 (v/v in PBS) was used to wash the plates thrice for $5 \mathrm{~min}$ to flush any residual 4\% paraformaldehyde. Subsequently, Actin-Tracker Green was diluted in PBS in a ratio of 1:40 as the working solution, which containing 5\% FBS (v/v) and 1\% Triton X-100 (v/v). Each well was supplemented with $300 \mu \mathrm{l}$ working solution, and incubated at $37^{\circ} \mathrm{C}$ without light for $45 \mathrm{~min}$. The working solution was then poured out and the excess solution containing PBS and $0.1 \%$ Triton X-100 (v/v) was removed by washing thrice (5 min each). Subsequently, either the AC16 cells or H9c2 cells were observed under a Nikon inverted microscope (Nikon Corp.) equipped with a Polaroid digital
Table I. Reaction system of first-strand cDNA synthesis.

\begin{tabular}{lc}
\hline Reagent & Volume $(\mu \mathrm{l})$ \\
\hline 5X PrimeScript Buffer (for real-time) & 2 \\
PrimeScriptRT Enzyme Mix I & 0.5 \\
Oligo dT Primer $(50 \mu \mathrm{M})$ & 0.5 \\
Random 6 mers $(100 \mu \mathrm{M})$ & 0.5 \\
Total RNA & $\mathrm{X}$ \\
RNase Free $\mathrm{dH}_{2} \mathrm{O}$ & $6.5-\mathrm{x}$ \\
Total volume per rxn & 10 \\
\hline
\end{tabular}

camera and photographed at x200 magnification. A total of 5 random images from each well were captured, and 10 individual cell surface areas per photography were measured by ImageJ software (National Institutes of Health).

$R T-q P C R$. Total RNA was isolated from the AC16 cells using TRIzol reagent (Invitrogen; Thermo Fisher Scientific, Inc.) according to the manufacturer's instructions. The concentration and purity of the total RNA were determined using a NanoDrop 2000c Spectrophotometer (Thermo Fisher Scientific, Inc.) and absorbance readings at $260 \mathrm{~nm}$ and $260 / 280 \mathrm{~nm}$, respectively, which were used as indicators of RNA concentration and purity. First-strand complementary DNA (cDNA) synthesis was performed with the PrimeScript ${ }^{\mathrm{TM}}$ RT Reagent kit (Perfect Real Time) in the reaction system presented in Table $\mathrm{I}$, and the reaction temperature was set as indicated in the manufacturer's protocol. Subsequently, using TB Green ${ }^{\mathrm{TM}}$ Advantage ${ }^{\circledR}$ qPCR Premix, the obtained cDNA and the homologous primers [as presented in Table II (41)] were mixed at the ratio indicated in Table III) in 96-well optical reaction plates (04729692001, Roche) and analyzed on a LightCycler 96 system (05815916001, Roche Diagnostics) according to the manufacturer's protocol. The reaction conditions were set as presented in Table IV. Each gene was analyzed in 3 parallel wells in each experiment, and the difference in incorporation data ( $\mathrm{Cq}$ values) from parallel wells should be $<1$. The results of ANP, BNP and $\beta$-MHC were normalized to those of GAPDH within the same sample and quantified by the formula $2^{-\Delta \Delta \mathrm{Cq}}(42)$ relative to the control group.

Western blot analysis. Either the AC16 cells or H9c2 cells were lysed with RIPA lysis buffer and the total protein content was analyzed with a BCA kit. Western blot analysis was performed as previously described (43). Briefly, equal amounts of total protein were separated by $10-15 \%$ SDS-PAGE and then transferred onto PVDF membranes (EMD Millipore). The membranes were blocked with $5 \%$ non-fat milk solution for $1 \mathrm{~h}$ at room temperature and incubated overnight at $4^{\circ} \mathrm{C}$ with primary antibodies against GAPDH $(1: 3,000)$, ANP $(1: 2,000)$, H3 (1:3,000), NF-кBIA $(1: 2,000)$, or p65 $(1: 2,000)$. The membranes were then washed 3 times with TBS-Tween-20 and further incubated with HRP-conjugated secondary antibodies $(1: 5,000)$ at room temperature for $1 \mathrm{~h}$. The membranes with immune complexes were visualized by a chemiluminescence 
Table II. Primer sequences used for RT-qPCR assays (41).

Primers

\begin{tabular}{lll}
\cline { 2 - 3 } Gene & \multicolumn{1}{c}{ Forward (5' to $\left.3^{\prime}\right)$} & Reverse (5' to 3') \\
\hline ANP & CAGCAAGCAGTGGATTGCTCCT & TCTGCGTTGGACACGGCATTGT \\
BNP & TGGAAACGTCCGGGTTACAGGA & TCCGGTCCATCTTCCTCCCAAA \\
GAPDH & GGGCAAAGGCAAGGCCAAGAAA & ATGGGTGGAGCGCAAGTTGGTCA \\
\hline
\end{tabular}

ANP, atrial natriuretic peptide; BNP, brain natriuretic peptide; $\beta$-MHC, $\beta$-myosin heavy chain.

Table III. Reaction system used for qPCR.

\begin{tabular}{lc}
\hline Reagent & Volume $(\mu \mathrm{l})$ \\
\hline TB Green Advantage qPCR Premix & 10 \\
Forward primer $(10 \mu \mathrm{M})$ & 0.4 \\
Reverse primer $(10 \mu \mathrm{M})$ & 0.4 \\
Rnase Free $\mathrm{dH}_{2} \mathrm{O}$ & 7.2 \\
cDNA sample & 2 \\
Total volume per rxn & 20 \\
\hline
\end{tabular}

imaging system (ImageQuant LAS 4000 Mini; GE Healthcare Life Sciences). The intensity of the bands was measured by ImageJ software (National Institutes of Health) and analyzed with IBM SPSS Statistics 23.0 (IBM Corp.).

ROS detection and quantification. Using the Reactive Oxygen Species Assay kit, the intracellular ROS levels in the PQQ-pre-treated and/or Iso-treated groups were detected, and the changes in the mean fluorescence intensity were quantified by ImageJ software. The experimental procedure was performed according to the manufacturer's instructions. In brief, the cells were loaded with dichloro-dihydro-fluorescein diacetate probes at a ratio of 1:1,000 (v/v) in $10 \mathrm{ml}$ serum-free medium and were incubated at room temperature for $30 \mathrm{~min}$. The groups were set as described above in the section entitled 'Cell culture and grouping'. The cells were then washed thrice for $15 \mathrm{~min}$ with serum-free medium. Images were obtained at x200 magnification with a Nikon inverted microscope (Nikon Corp.) equipped with a charge-coupled device camera (Hamamatsu). Each well was photographed in 5 random fields and analyzed by Image J software as described above in the section entitled 'Cell morphometric analysis'. Moreover, the ROS levels were also analyzed by analytical flow cytometry (BD Biosciences) at an excitation wavelength of $488 \mathrm{~nm}$ and an emission wavelength of $525 \mathrm{~nm}$, respectively. Flowjo software (Flowjo, LLC) was used to analyze the results of flow cytometry.

Mitochondrial membrane potential (MMP) detection. As JC-1 is an ideal fluorescent probe for detecting MMP, the change in fluorescent color by the JC-1 probe was obtained to detect the change in MMP (44). The JC-1 probe (Mitochondrial
Table IV. Reaction conditions for RT-qPCR assays.

\begin{tabular}{lcrr}
\hline Procedure & $\begin{array}{r}\text { Temperature } \\
\left({ }^{\circ} \mathrm{C}\right)\end{array}$ & $\begin{array}{c}\text { Duration } \\
(\mathrm{sec})\end{array}$ & Cycle \\
\hline Initial denaturation & 95 & 600 & 1 \\
Denaturation & 95 & 15 & 45 \\
Annealing & 60 & 34 & \\
\hline
\end{tabular}

membrane potential assay kit with JC-1, C2006, Beyotime Institute of Biotechnology) was used to detect changes in MMP in the AC16 cells treated with Iso and/or PQQ pre-treatment. The experimental procedure was performed as previously described $(45,46)$. The fluorescence images were obtained using a fluorescent microscope (Nikon Corp.).

Statistical analysis. IBM SPSS Statistics 23.0 (IBM Corp.) was used for statistical analysis. All data are presented as the means \pm standard deviation. Differences between 2 groups were analyzed with an unpaired Student's t-test. Statistical analysis among various groups was conducted by one-way analysis of variance with Tukey's post hoc test. $\mathrm{P}<0.05$ was considered to indicate a statistically significant difference.

\section{Results}

$P Q Q$ prevents Iso-induced hypertrophy in mice. The results obtained are presented in Fig. 1, which illustrates that the cell morphological changes in the mouse cardiac muscle in the Iso-treated C57 mice. The surface area increased significantly, while following pre-treatment with PQQ, the increase in the surface area was reduced (Fig. 1A and B). Moreover, the ratio of heart weight/body weight in the Iso group was higher than that in the control group. In the PQQ + Iso group, a decrease in the ratio of heart weight/body weight was observed compared to the Iso group (Fig. 1C). These results indicated that PQQ exerted an inhibitory effect on ISO-induced cardiac hypertrophy in vivo.

Effects of Iso and PQQ on the viability of AC16 cells. To examine the effects of Iso and PQQ on AC16 cells, cell viability was determined with a rapid and sensitive CCK- 8 assay kit according to the manufacturer's instructions. The OD values, 
A
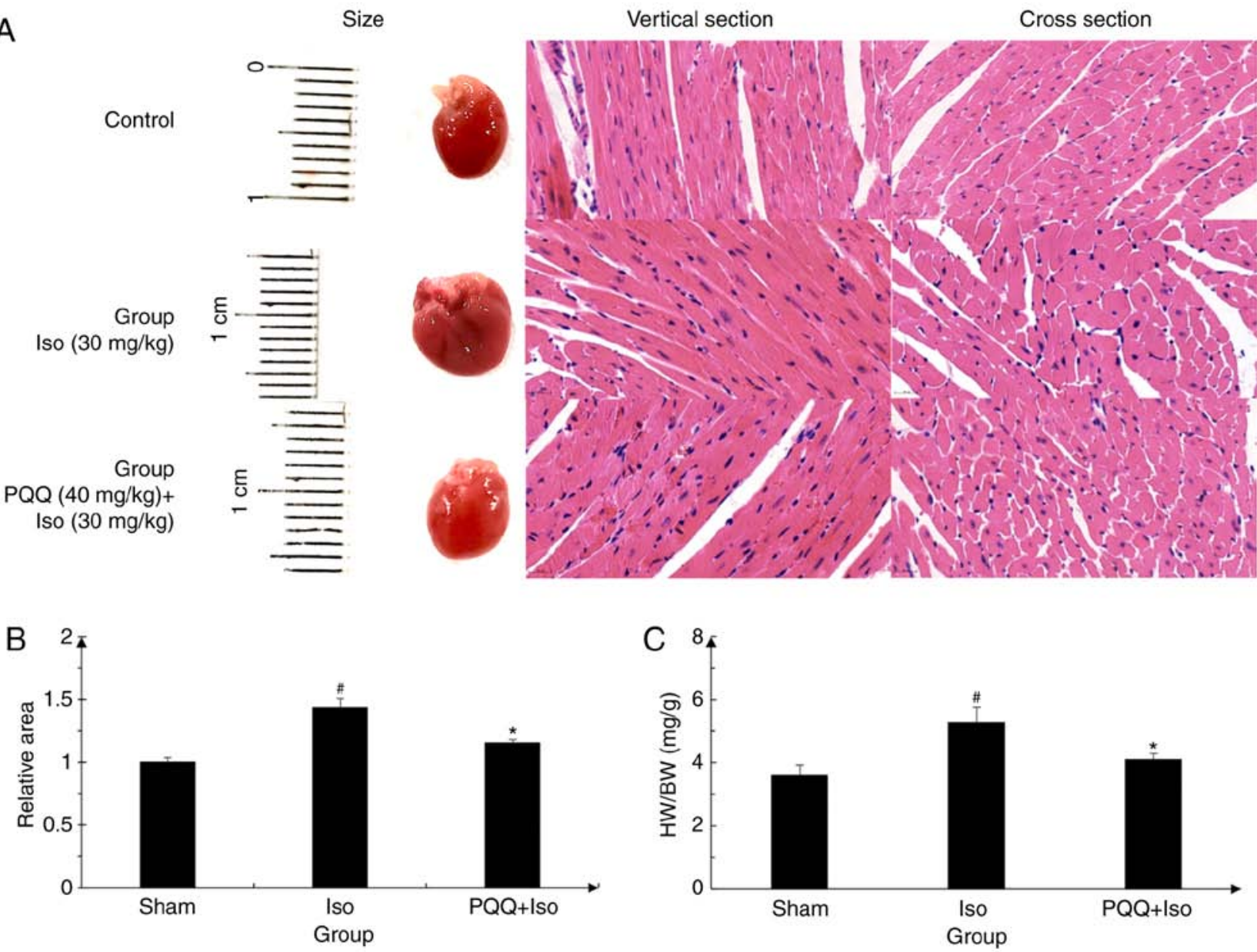

Figure 1. Effect of PQQ against Iso-induced cardiac hypertrophy. (A) Hematoxylin and eosin (H\&E) staining of mouse heart sections in the control group, Iso group and PQQ + Iso group after 5 weeks of intraperitoneal injection. (B) The C57 mice cardiac muscle cells surface area was quantitated by ImageJ software and the control group was set as 1 . (C) Heart weight $(\mathrm{HW}) /$ body weight $(\mathrm{BW})$ ratio. Data are presented as the means $\pm \mathrm{SD}(\mathrm{n}=6) .{ }^{*} \mathrm{P}<0.05$ vs. the control group, ${ }^{*} \mathrm{P}<0.05$ vs. the Iso group. $\mathrm{PQQ}$, pyrroloquinoline quinone; Iso, isoproterenol hydrochloride.

which directly reflect the survival status and viability of the AC16 cells, were 0.2-0.4 for the Iso and PQQ groups of AC16 cells (Fig. 2A and C). AC16 cell viability for the control well was set at $100 \%$, and relatively, the other well percentages were calculated. The results of the CCK- 8 assay demonstrated that concentrations of Iso from 5 to $160 \mu \mathrm{M}$ and those of PQQ from 1 to $200 \mu \mathrm{M}$ had no significant effect on AC16 cell viability (Fig. 2B and D). Thus, subsequent studies that could identify alterations in gene expression would not lead to changes in cell viability or cytotoxicity. Based on the above findings and those of previous studies $(22,47)$, the concentration of $20 \mu \mathrm{M}$ was selected as the therapeutic concentration of Iso.

$P Q Q$ acts against Iso-induced hypertrophy. The increase in AC16 cell size can directly reflect the progression of cardiac hypertrophy. This study found that the Iso-treated AC16 cells exhibited a marked change in morphology and their surface area increased significantly, while the increase in the surface area was reduced following pre-treatment of the AC16 cells with PQQ (Fig. 3A and B). With $1 \mu \mathrm{M}$ PQQ pre-treatment, the surface area of Iso-induced cardiomyocyte hypertrophy was significantly reduced compared with that of the Iso group without PQQ pre-treatment, and the increased surface area was reduced by $29 \%$. The $2.5 \mu \mathrm{M}$ PQQ pre-treated group exhibited a $38 \%$ decrease in cell surface area compared with that of the Iso group, while the $5 \mu \mathrm{M}$ PQQ pre-treated group exhibited a decrease of $53 \%$ in cell size compared with that of the Iso group, and the cell surface area was almost unaltered macroscopically compared with that of the control group (Fig. 3A and B). To further investigate the protective effects of PQQ on the heart, the effects of PQQ on H9c2 cells with Iso-induced hypertrophy were also detected (Fig. 3C and D), and the results revealed a consistent trend with what was observed with the AC16 cells.

The present study examined the mRNA expression levels of the hypertrophic markers, ANP, BNA and $\beta$-MHC, in AC16 cardiomyocytes relative to those of the blank control group in order to verify whether PQQ can protect against Iso-induced cardiac hypertrophy. The results (Cq value) of ANP, BNP and $\beta$-MHC were normalized to those of GAPDH in the same sample and were quantified by the formula $2^{-\Delta \Delta C q}$. The expression of ANP, BNP and $\beta$-MHC in the Iso-treated group increased by 1.42-, 1.71- and 1.74-fold vs. the control group at $24 \mathrm{~h}$. Upon pre-treatment with PQQ, the expression of hypertrophic markers decreased. Following pre-treatment with $1 \mu \mathrm{M}$ PQQ, the expression levels of ANP, BNP and $\beta$-MHC were downregulated, and the downregulation of BNP and $\beta$-MHC was statistically significant, while the differ- 

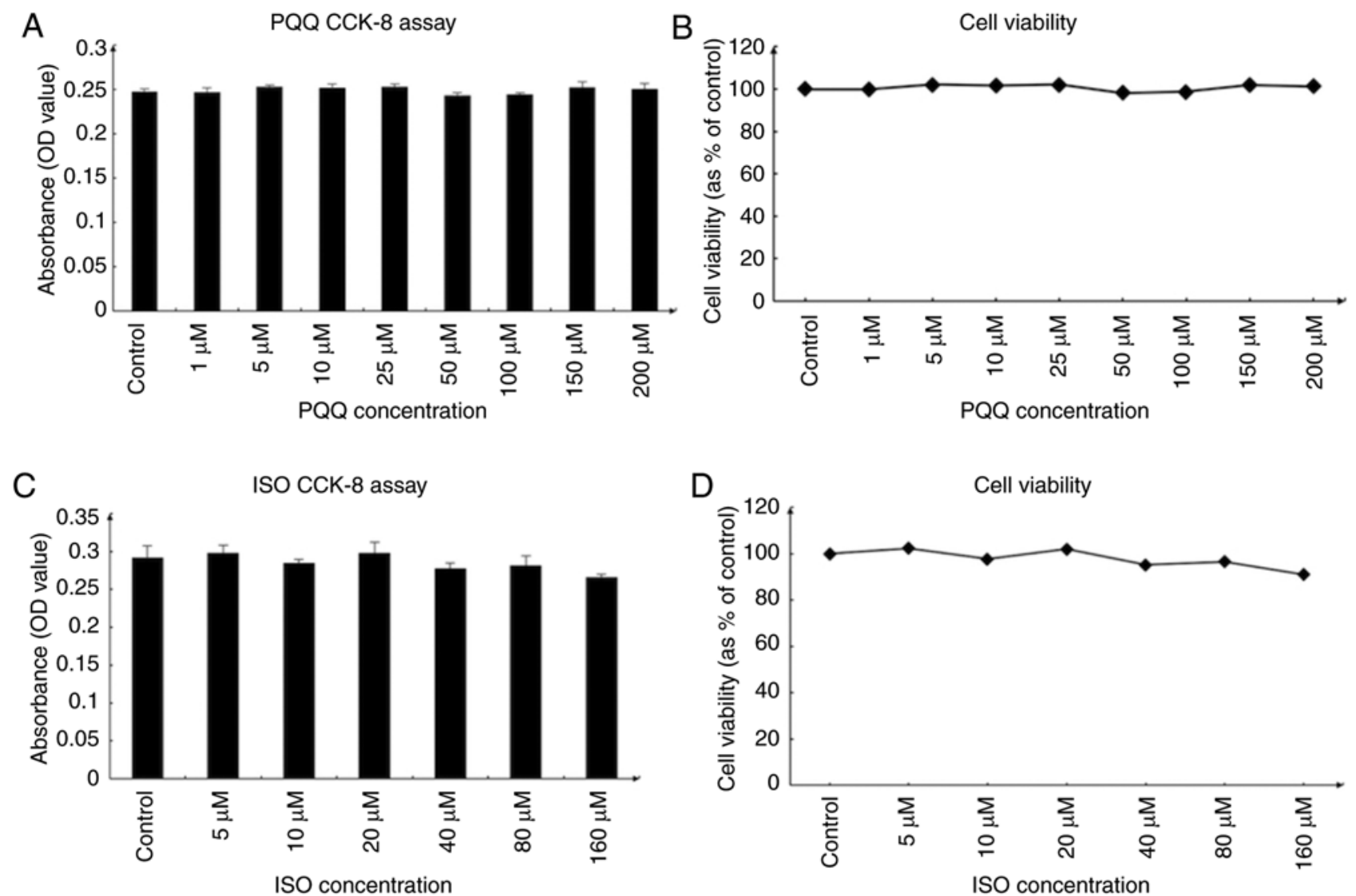

Figure 2. Effect of Iso on the viability of AC16 cells. (A) Changes in the optical density value of AC16 cells treated with increasing concentrations of PQQ (B) Viability of AC16 cells treated with various concentrations of PQQ. (C) Changes in the optical density value of AC16 cells treated with increasing concentrations of Iso. (D) Viability of AC16 cells treated with various concentrations of Iso. The viability of AC16 cells in the control group was set at 100 , and the data on the line chart represent the percentage of the control group. Data are presented as the means \pm SD ( $n=3$ ). PQQ, pyrroloquinoline quinone; Iso, isoproterenol hydrochloride.

ence in ANP was not statistically significant. Pre-treatment with a higher concentration of PQQ (i.e., 2.5 and $5 \mu \mathrm{M}$ ) led to a downregulation in the expression levels of ANP, BNP and $\beta$-MHC, which was more significant with increasing concentrations. The levels of these markers in the $5 \mu \mathrm{M}$ PQQ-pre-treated group were similar to those of the control group (Fig. 4A).

To further examine the effect of PQQ on cardiac hypertrophy, the present study also detected the changes in ANP protein expression by western blot analysis. ANP expression in the Iso-treated group was significantly upregulated. Upon pre-treatment with various concentrations of PQQ $(1,2.5$ and $5 \mu \mathrm{M})$, ANP protein expression was decreased compared with that of the Iso-treated group. Although the difference in the ANP protein level between the Iso-treated group and the $1 \mu \mathrm{M}$ PQQ-pre-treated group was not statistically significant, the trend in the downregulation of ANP protein with the increasing PQQ concentration was consistent with the trend observed for ANP mRNA expression (Fig. 4B and C). Overall, these findings indicated that PQQ pre-treatment attenuated Iso-induced hypertrophy, and the protective effects of PQQ were more effective as the concentration increased from 1 to $5 \mu \mathrm{M}$. Furthermore, ANP expression was also detected in the $\mathrm{H} 9 \mathrm{c} 2$ cells. The results revealed that ANP protein expression in the Iso-treated group was significantly upregulated, and the protective effects of $\mathrm{PQQ}$ were enhanced as the concentration increased, which was in accordance with the results obtained for the AC16 cells (Fig. 4D and E).

$P Q Q$ prevents the Iso-induced hypertrophy of ACl6 cells via the inhibition of the $N F-\kappa B$ signaling pathway. Under normal physiological conditions, NF- $\kappa B$ in the cytoplasm and NF- $\kappa B$ inhibitor (I $\kappa \mathrm{B})$ are combined and remain in an inactive state. However, under inflammatory or other stimulating conditions,

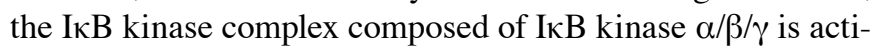
vated to promote serine phosphorylation at positions 32 and 36 of the I $\kappa \mathrm{B} \alpha$ protein, leading to the degradation of I $\kappa \mathrm{B} \alpha$ by ubiquitination and the release of free NF- $\kappa B$. This free NF- $\kappa B$ can enter the nucleus binding with the specific site, and can subsequently mediate a series of cellular responses, such as inflammation through transcriptional activation of its target gene (48). NF- $\kappa$ BIA is one of the proteins of I $\kappa$ B that can

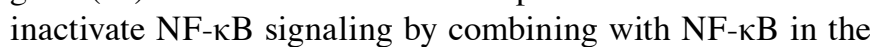
cytoplasm. The present study thus analyzed the expression of NF- $\kappa B I A$ and nuclear p65 by extracting nuclear proteins to determine the mechanism through which the NF- $\kappa B$ signaling pathway participates in cardiac hypertrophy induced by Iso or by treatment with PQQ.

As shown in Fig. 5, nuclear p65 expression in the Iso pre-treated group increased by 1.5 -fold vs. the control group, while the expression of NF-кBIA decreased by $60 \%$. Following pre-treatment with PQQ, the expression of nuclear 


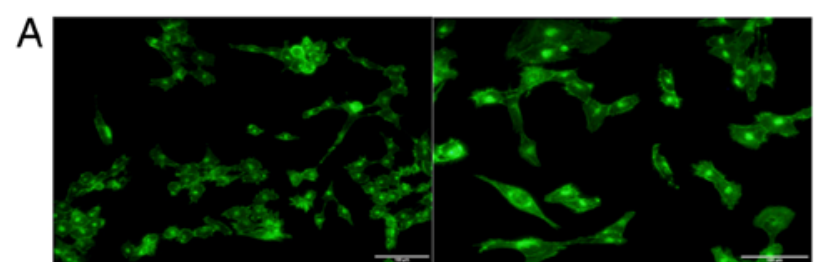

Control

Iso

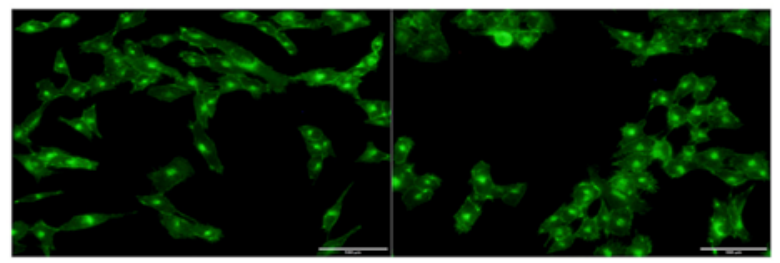

$1 \mu \mathrm{M} P Q Q+\mathrm{Iso}$

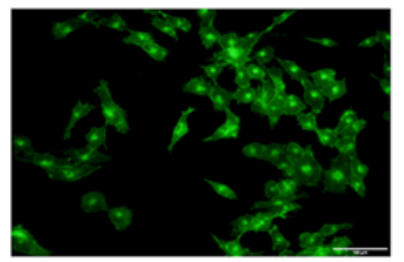

$5 \mu \mathrm{M} P Q Q+l s o$

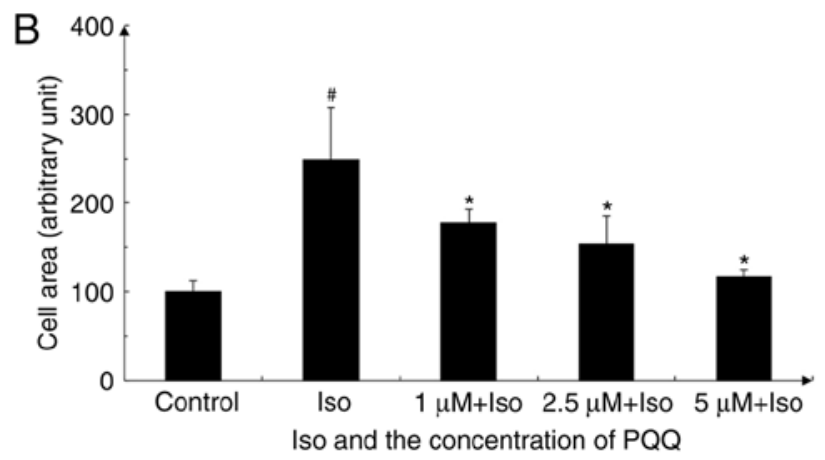

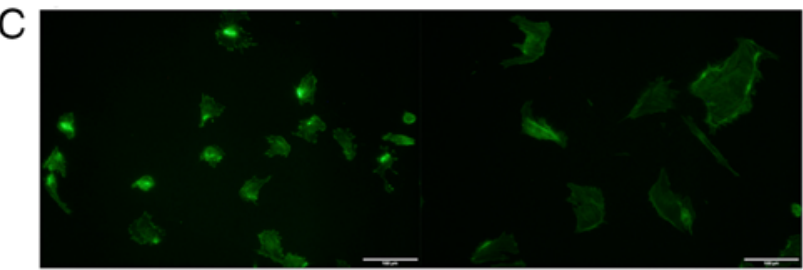

Control

Iso

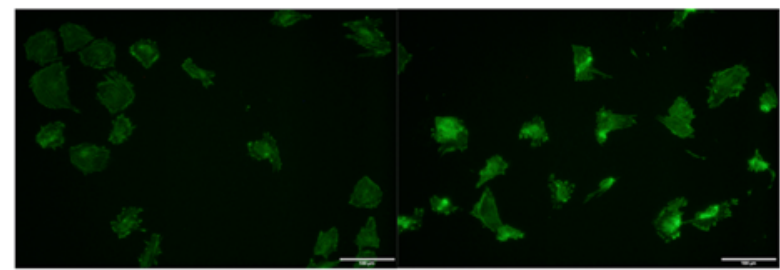

$1 \mu \mathrm{M} P Q+\mathrm{Iso}$

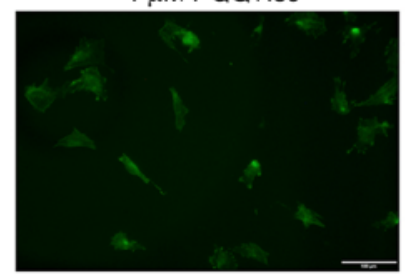

$5 \mu \mathrm{M} P Q++\mathrm{so}$

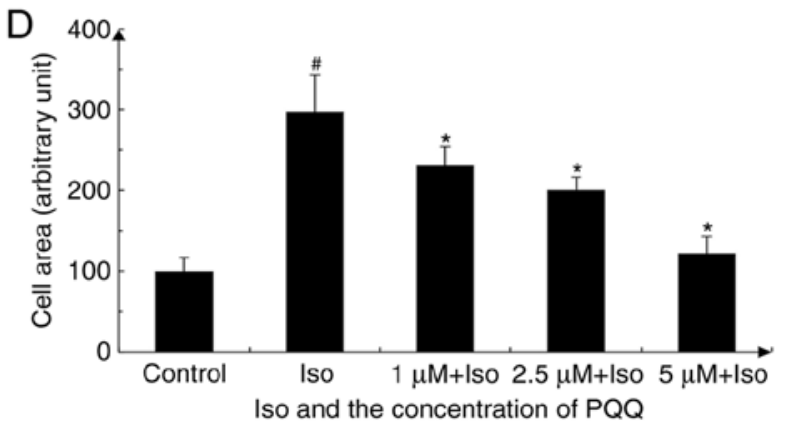

Figure 3. PQQ acts against Iso-induced hypertrophy in AC16 cells or in H9c2 cells. (A) After being treated with various concentrations of PQQ or 20 $\mu \mathrm{M}$ Iso (the groups were set as described in the 'Materials and methods', and the change in the size of AC16 cells was photographed. The morphology of the AC16 cells was evaluated with the Actin-Tracker Green kit. (B) The area of AC16 cells was quantitated by ImageJ software. The control group was set at 100, and the other groups represent a percentage of the control. (C) Following treatment with various concentrations of PQQ or $20 \mu \mathrm{M}$ Iso (groups were set as described in the 'Materials and methods'), the rat H9c2 cell size changed and was photographed. The morphology of the rat H9c2 cells was examined by the Actin-Tracker Green kit. (D) The rat H9c2 cells area were quantified by ImageJ software and the control group was set at 100, the other groups are presented as a percentage of the control. ${ }^{\#} \mathrm{P}<0.05$ vs. the control group, ${ }^{*} \mathrm{P}<0.05$ vs. the Iso group. $\mathrm{PQQ}$, pyrroloquinoline quinone; Iso, isoproterenol hydrochloride.

p65 in the $1 \mu \mathrm{M}$ PQQ group was decreased by $33 \%$ compared with that of the Iso pre-treated group, and it was significantly decreased with the increasing concentrations of PQQ. By contrast, NF- $\kappa \mathrm{BIA}$ expression significantly increased with the increasing concentrations of PQQ (Fig. 5B). Following treatment with Iso, the $\mathrm{NF}-\kappa \mathrm{B}$ translocation to the nucleus significantly increased, while with the increasing concentrations of PQQ, the protective effects of PQQ were enhanced, as indicated by the significant proportionate decrease of $\mathrm{NF}-\kappa \mathrm{B}$ translocation to the nucleus (Fig. 5). The above-mentoined results indicated that, by inhibiting the $\mathrm{NF}-\kappa \mathrm{B}$ signaling pathway, PQQ may inhibit NF- $\kappa \mathrm{B}$ from entering the nucleus, thus acting against Iso-induced hypertrophy in AC16 cells.

$P Q Q$ reduces $R O S$ levels in AC16 cells with Iso-induced hypertrophy. It has been reported that ROS accumulation is closely associated with inflammation (49), and PQQ effectively scavenges the production of intracellular ROS, as demonstrated in previous studies (50-52). The changes in ROS levels in AC16 cells with Iso or PQQ treatment were thus detected and photographed and quantified with ImageJ software (Fig. 6). As observed, the fluorescence intensity of the Iso pre-treated group was markedly stronger than that of the control and PQQ-treated groups (Fig. 6A). Upon treatment with Iso, the ROS levels were significantly increased by 2.2-fold relative to the control group, whereas the ROS levels of the group pre-treated with 1,2.5 and $5 \mu \mathrm{M}$ PQQ decreased by 18,27 and $50 \%$ compared with those of the Iso pre-treated group (Fig. 6B). Moreover, the changes in the intracellular fluorescence intensity of the AC16 cells were detected by flow cytometry and the results revealed a consistent trend with the results of the images (Fig. 6C and D).

$P Q Q$ increases MMP in AC16 cells with Iso-induced hypertrophy. During the process of respiratory oxidation, the mitochondria store the energy generated by electrochemical 

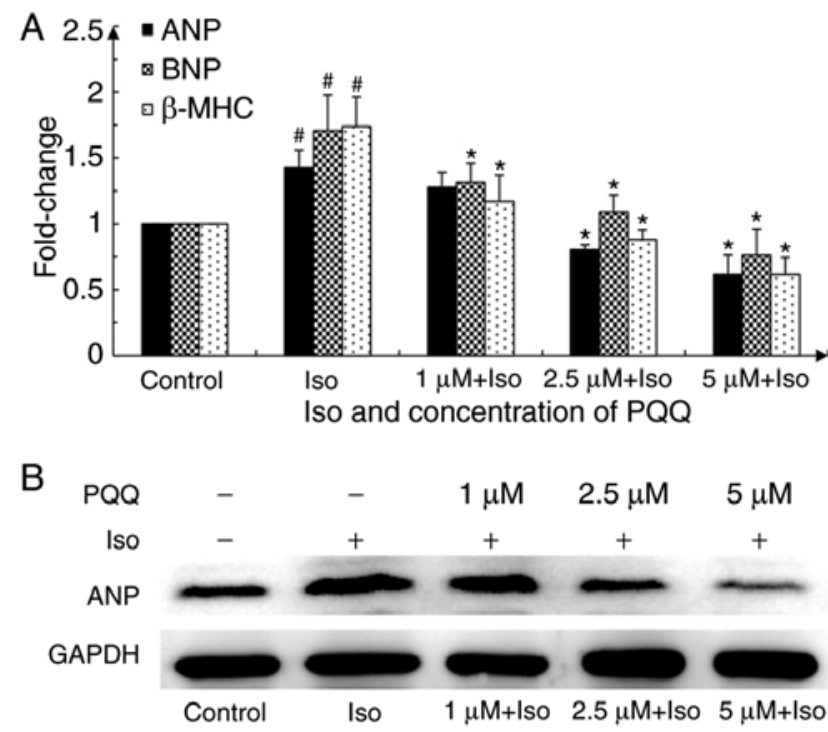

C
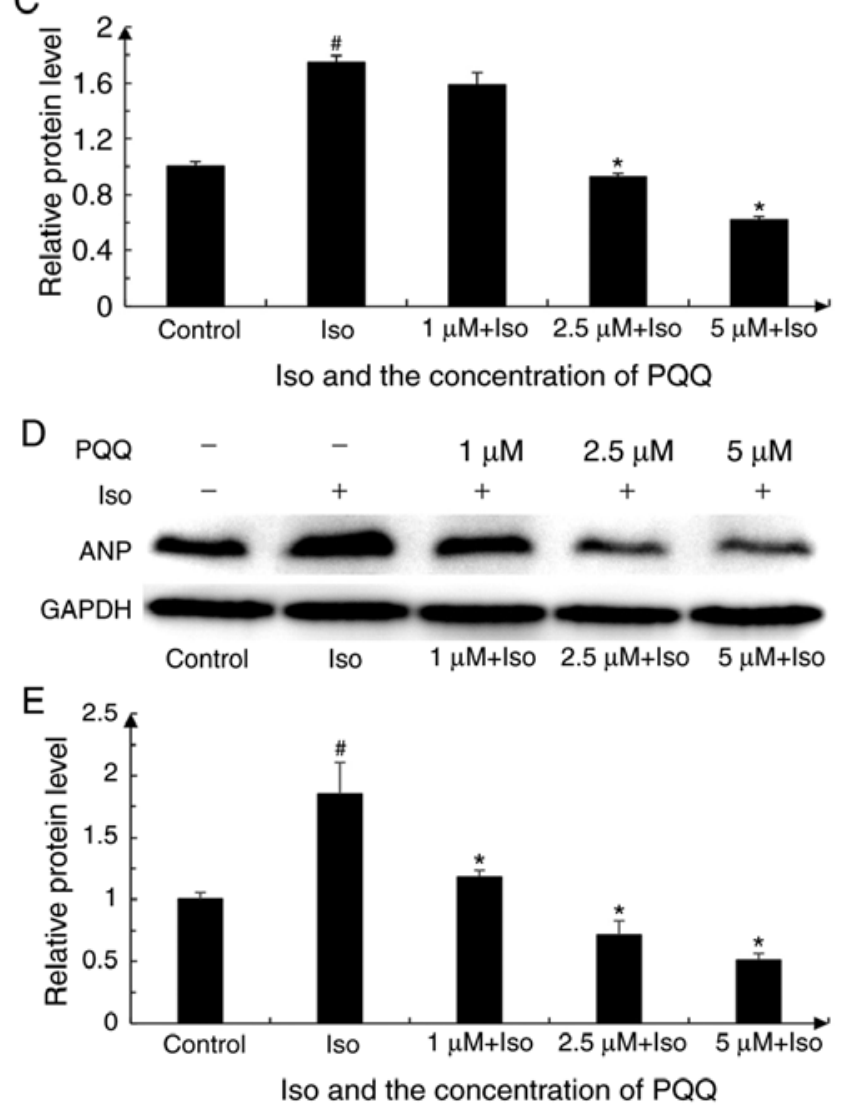

Figure 4. Effect of PQQ against Iso-induced hypertrophy in AC16 cells is reflected by the changes in cardiac hypertrophic markers. (A) Changes in cardiac hypertrophic markers mRNA levels (groups were set as described in the 'Materials and methods'). The data represent the mean of 3 parallel wells in 3 independent replicate experiments. The control group was set as 1 . (B) Changes in protein expression of the cardiac hypertrophy marker, ANP, upon pre-treatment with various concentrations of PQQ or Iso in AC16 cells. GAPDH was used as an internal reference for total protein. The western blot is representative of 3 independent replicate experiments that yielded similar results. (C) Expression of ANP was quantified by ImageJ software. The control group was set as 1. (D) Changes in the protein expression of the cardiac hypertrophy marker, ANP, in $\mathrm{H} 9 \mathrm{C} 2$ cells pre-treated with various concentrations of PQQ or treated with Iso. GAPDH was used as an internal reference protein of total protein. The western blot represents 1 of 3 independent replicate experiments that yielded similar results. (E) Expression of ANP was quantified by ImageJ software and we set the control group as $1 .{ }^{*} \mathrm{P}<0.05$ vs. the control group, ${ }^{*} \mathrm{P}<0.05$ vs. the Iso group. $\mathrm{PQQ}$, pyrroloquinoline quinone; Iso, isoproterenol hydrochloride; ANP, atrial natriuretic peptide.
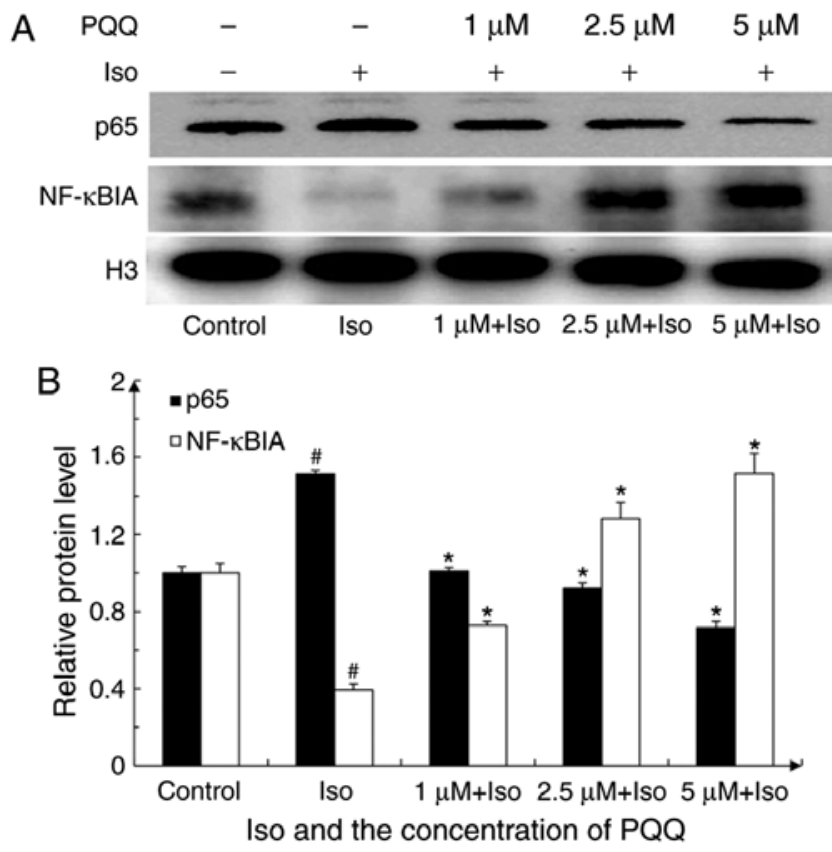

Figure 5. Changes in NF-кBIA and nuclear p65 expression. (A) Changes in NF-кBIA and p65 following treatment with various concentrations of PQQ or Iso (groups were set as described in the 'Materials and methods'). H3 was used as an internal reference for both nuclear protein and total protein. Shown is a representative western blot of 3 independent replicate experiments that yielded similar results. (B) Expression of NF-кBIA, p65 and H3 was quantified by ImageJ software. The control group was set as 1 . ${ }^{\text {}} \mathrm{P}<0.05$ vs. the control group, ${ }^{*} \mathrm{P}<0.05$ vs. the Iso group. $\mathrm{PQQ}$, pyrroloquinoline quinone; Iso, isoproterenol hydrochloride; H3, histone-3.

potential energy in the mitochondrial inner membrane, causing asymmetric distribution of proton and other ion concentrations on both sides of the inner membrane to form $\mathrm{MMP}$ (53), which is a key parameter for evaluating mitochondrial function $(54,55)$. The results obtained in this study for MMP are presented in Fig. 7. The red fluorescence intensity of JC-aggregates in the control group was significantly higher than that of JC-1monomers and the 'Merge' image synthesized by the two panels is dominated by the red fluorescence of JC-aggregates. The green fluorescence intensity of JC-1 monomers in the AC16 cells in the Iso group was significantly higher than that of the control group; the synthesized 'Merge' image is mainly compiled of green fluorescence. The red fluorescence of JC-aggregates in the group pre-treated with $1 \mu \mathrm{M}$ PQQ was higher than that in the Iso group, and the 'Merge' image red fluorescence and the green fluorescence are almost the same, and the 'Merge' image is colored orange. The green fluorescence in the group pre-treated with $2.5 \mu \mathrm{M}$ PQQ was weakened, and the red fluorescence in the synthesized 'Merge' image is greater than that in the Iso group. The green fluorescence in the group pre-treated with $5 \mu \mathrm{M}$ PQQ was significantly weaker than that in the Iso group, and the red fluorescence in the 'Merge' image is greater than that in the Iso group, but slightly weaker than that in the control group (Fig. 7).

\section{Discussion}

Cardiomyocytes of the adult human heart almost lose their proliferative capacity; thus, the heart does not regenerate significant amounts of lost tissue following injury, and is not 


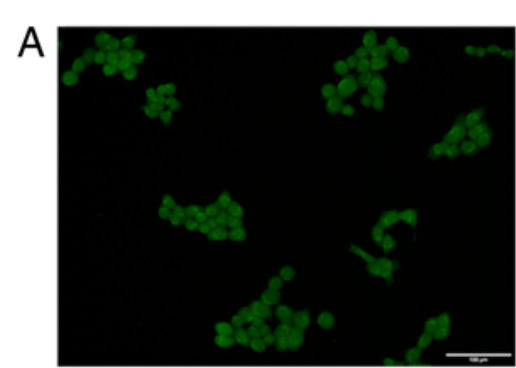

Control

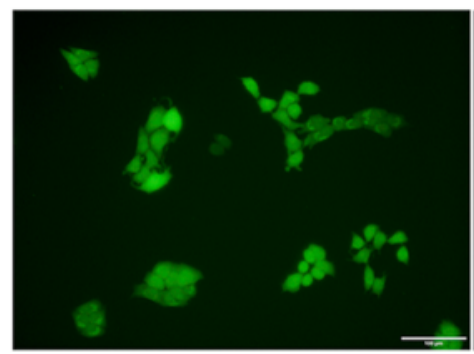

$1 \mu \mathrm{M} P Q Q+I s o$

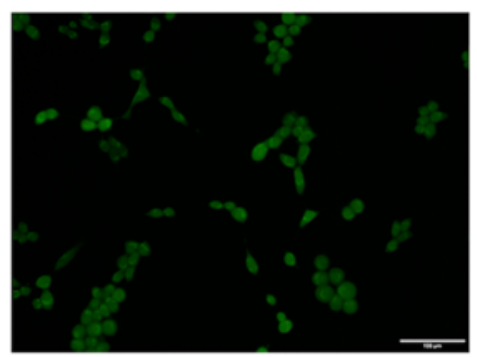

$5 \mu \mathrm{M} P Q Q+I s o$

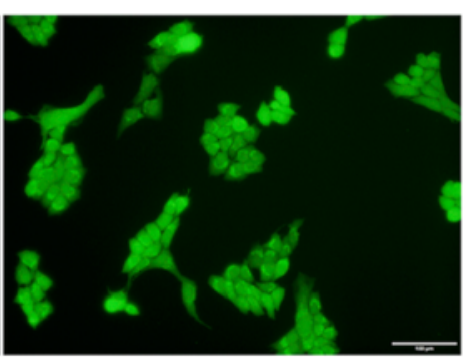

Iso

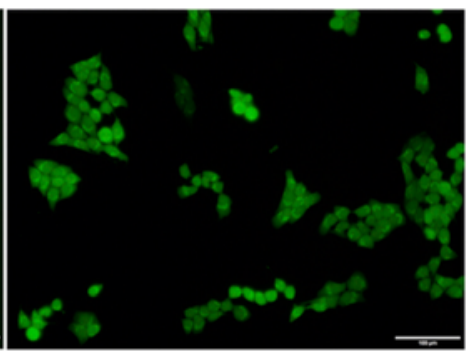

$2.5 \mu \mathrm{M} P Q Q+I s o$

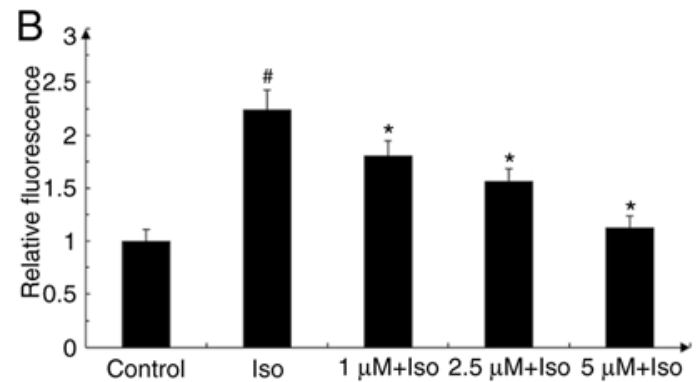

Iso and the concentration of PQQ

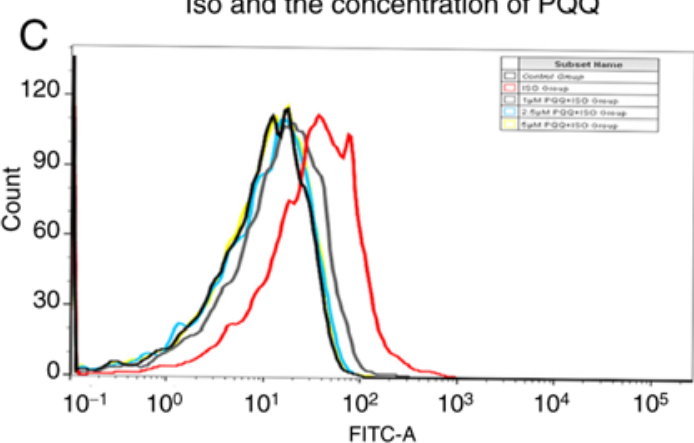

$\mathrm{D}$

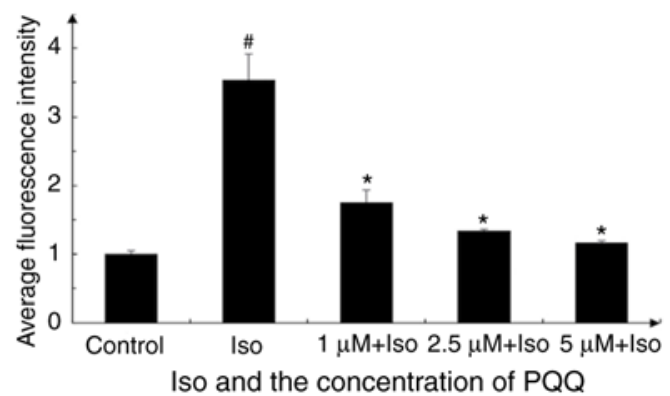

Figure 6. PQQ alters the levels of ROS in AC16 cells. (A) Effect of PQQ on Iso-induced ROS levels in AC16 cells was detected with the Reactive Oxygen Species Assay kit and then photographed (groups were set as described in the 'Materials and methods'). (B) The levels of ROS were quantified by ImageJ software, with the mean fluorescence intensity of the control group set as 1. (C) DCFH-DA fluorescent dye was used to detect ROS production in the AC16 cells with a flow cytometer. PQQ attenuated Iso-induced ROS levels in AC16 cells. Black line, control group; red line, Iso group; gray line, $1 \mu \mathrm{M}$ PQQ + Iso group; blue line, $2.5 \mu \mathrm{M}$ PQQ + Iso group; yellow line, $5 \mu \mathrm{M}$ PQQ + Iso group. (D) Statistical data are presented based on experiments performed in triplicate. Data are presented as the means $\pm \mathrm{SD}(\mathrm{n}=3)$. ${ }^{\#} \mathrm{P}<0.05$ vs. the control group, ${ }^{*} \mathrm{P}<0.05$ vs. the Iso group. PQQ, pyrroloquinoline quinone; Iso, isoproterenol hydrochloride; ROS, reactive oxygen species.

able to create new, functional muscle following scarring and hypertrophy, which indicates that myocardial damage may be irreversible $(56,57)$. Therefore, protective measures are often ineffective in the end stages of cardiovascular disease. Cardiac hypertrophy occurs at the initial stage of heart failure development. If this could be prevented, and the compensatory period of cardiac hypertrophy could be protected to restore the function of cardiomyocytes, heart failure could be effectively prevented. Therefore, the present study focused on the preventive effects of PQQ against cardiac hypertrophy.

PQQ is present in mammalian tissues and human milk, and has excellent antioxidant properties. It is similar to B vitamins, which are currently proposed to be classified as vitamins in Japan $(58,59)$. PQQ is widely used in industrial, agricultural and medical treatments (14). In clinical practice, PQQ has demonstrated protective effects in the liver and brain $(9,11)$, and it has been studied in relation to cardiovascular diseases. Previous studies have demonstrated that PQQ can attenuate the expression of matrix metalloproteinase-1 (MMP-1), matrix metalloproteinase-3 (MMP)-3, tumor necrosis factor- $\alpha$ (TNF- $\alpha$ ) and interleukin (IL)-6 in IL-1 $\beta$-treated SW982 cells by attenuating the activation of $\mathrm{NF}-\kappa \mathrm{B}$, and can also significantly improve the clinical symptoms of collagen-induced arthritis in mice (12). Previous in vitro studies have indicated that PQQ exerts significant anti-neuroinflammatory effects in microglial cells by regulating the $N F-\kappa B$ and $\mathrm{p} 38$ mitogen-activated protein kinase (MAPK) signaling pathways $(9,60)$.

In rats, high doses of PQQ $(15 \mathrm{mg} / \mathrm{kg}$ ) have been shown to reduce the myocardial infarct size and attenuate myocardial dysfunction and the levels of malondialdehyde/thiobarbituric acid reactive substances in myocardial tissue (17). These substances are often used as a measure of free radical-induced lipid peroxidation and oxidative stress. Moreover, the administration of low doses of PQQ ( $3 \mathrm{mg} / \mathrm{kg}$ ) or metoprolol at the beginning of reperfusion has been shown to be effective in reducing the myocardial infarct size, improving cardiac function and preventing mitochondrial dysfunction. At non-toxic doses, PQQ is superior to metoprolol in protecting mitochondria from oxidative damage and reducing lipid peroxidation (15). The above-mentioned results indicate that the effects of PQQ on protecting the heart from ischemia/reperfusion injury may be accomplished by its ability to scavenge free radicals to 


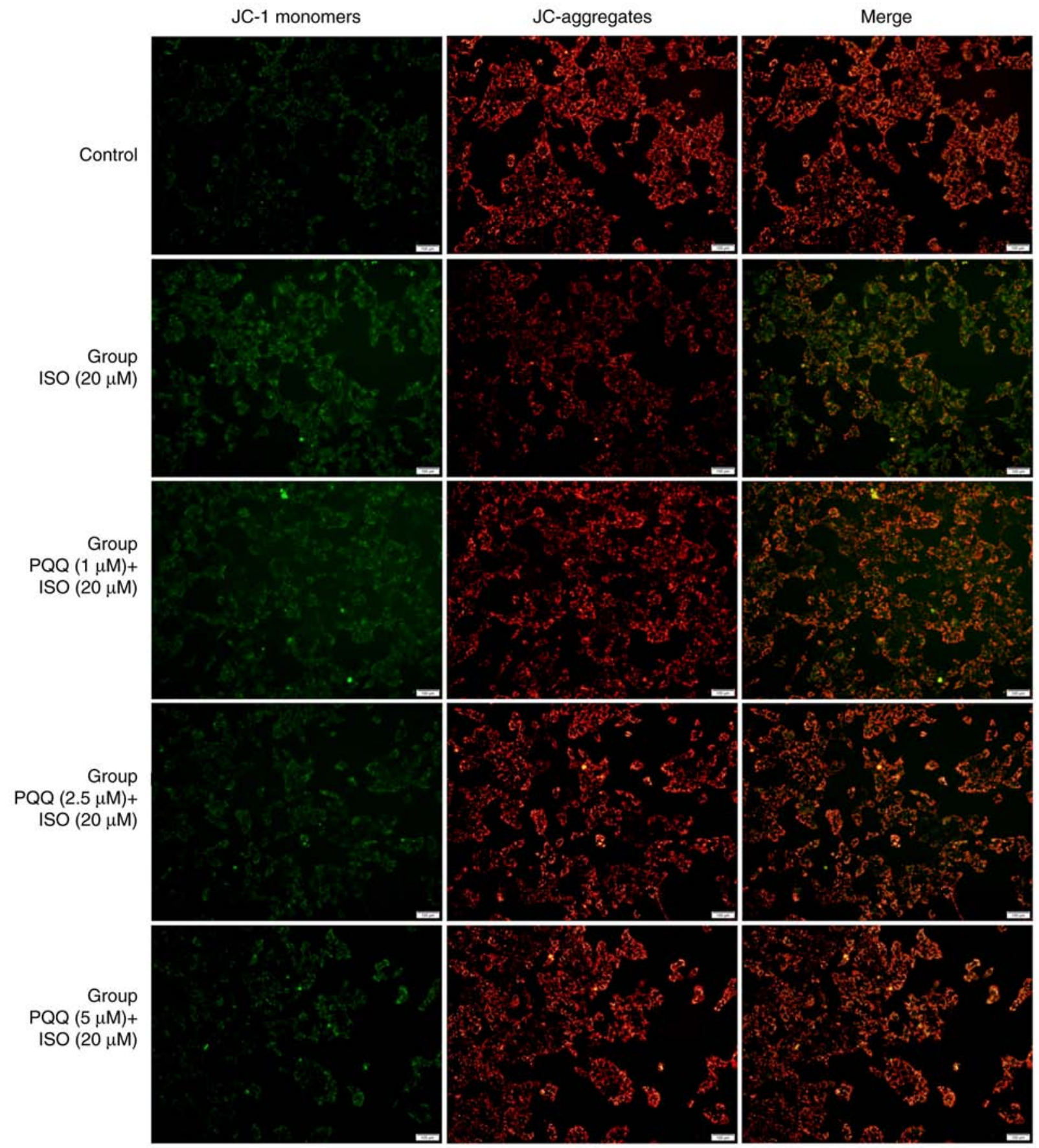

Figure 7. Effects of PQQ against Iso-induced changes in MMP in AC16 cells. The effects of PQQ on Iso-induced changes in MMP in AC16 cells were detected using the Mitochondrial membrane potential assay kit with JC-1 and were then photographed (groups were set as described in the 'Materials and methods'). PQQ, pyrroloquinoline quinone; Iso, isoproterenol hydrochloride; MMP, mitochondrial membrane potential.

protect the mitochondria from oxidative stress. In addition, it has been reported that the nanocurcumin-PQQ formulation prevents hypertrophy-induced pathological damage by relieving mitochondrial stress in cardiomyocytes under hypoxic conditions, while under these conditions, PQQ treatment alone can improve cellular viability (19).

As previously reported, Iso can promote the degradation and nuclear translocation of NF- $\kappa B$, thereby activating the NF- $\kappa \mathrm{B}$ signaling pathway (61). With the activation of NF- $\kappa B$, intracellular ROS levels are elevated, and the adaptive response of the heart to this involves a series of corresponding compensatory processes such as changes in gene expression, protein synthesis and the myocardial cell area, which ultimately leads to compensatory hypertrophy. The results of this study revealed that the ROS levels in the AC16 cells following PQQ pre-treatment were significantly lower than those in the Iso-treated group, and that PQQ decreased the expression of NF- $\kappa \mathrm{B}$ and inhibited its entry into the nucleus, suggesting that PQQ pre-treatment attenuated Iso-induced cardiac hypertrophy.

Previous studies have reported that the change in MMP is closely related to the accumulation of ROS, that a variety of cells with the accumulation of ROS under the action of different factors are accompanied by a decrease in MMP (62-64). 
Normal MMP is a prerequisite for maintaining oxidative phosphorylation of mitochondria and producing adenosine triphosphate. The stabilization of MMP is beneficial to maintain the normal physiological function of cells. The findings of this study demonstrated that PQQ pre-treatment increased the level of MMP to mitigate Iso-induced cardiac hypertrophy, which suggested that PQQ can retain the stability of MMP to protect cardiomyocytes.

In conclusion, this study, to the best of our knowledge, demonstrated for the first time that PQQ can act against Iso-induced cardiac hypertrophy by attenuating the activation of NF- $\kappa \mathrm{B}$ phosphorylation in vitro. In addition, this study verified the effects of PQQ on preventing myocardial hypertrophy in vivo. $\mathrm{PQQ}$ also significantly reduced the Iso-induced accumulation of ROS and increased the level of MMP in cardiac hypertrophic AC16 cells. These findings suggest that PQQ has great potential for use as a novel therapeutic agent that may aid in the treatment of cardiac hypertrophy caused by inflammatory reactions.

Despite this, there are still some limitations to this study. In the present study, the authors did not supplement the other two western blot analysis results of BNP and $\beta$-MHC in addition to ANP, as these two indicators should be routinely tested. The mRNA expression levels of hypertrophic markers, including ANP, BNP and $\beta$-MHC were examined and changes in the protein expression in combination of ANP (Fig. 4), as well as changes in cell characterization (observed by Actin-Green staining experiments; Fig. 2) were examined, which proved that Iso can cause AC16 cardiomyocyte hypertrophy, and that PQQ exerts a protective effect against Iso-induced cardiac hypertrophy.

Although AC16 cells are broadly used in cardiovascular diseases, such as cardiomyocyte hypertrophy and myocardial injury $(65,66)$, it would have greatly increased the clinical value of this study if the role of PQQ was compared between cardiomyocyte cell lines and primary human cardiac fibroblasts; however, the authors failed to obtain primary human cardiac fibroblasts for some inevitable reasons. Thus, future studies to resolve this issue and aiming to verify the results in original human heart fibroblasts are warranted.

Furthermore, this study only validated the effects of pre-treatment with PQQ; thus, thereby further studies exploring the therapeutic and restorative effects of PQQ in later stages and time points are warranted to confirm any long-term beneficial effects of PQQ.

\section{Acknowledgements}

The authors would like to thank members of the State Key Laboratory of arrhythmia (Dr Li Jue), and Dr Yanfei Li and Miss Yuan Ma for providing guidance with fluorescence microscopy, as well as Miss Jiawen Wu and Miss Yongjia $\mathrm{Hu}$ for providing assistance with the statistical analysis.

\section{Funding}

The present study was supported by grants from the Shanghai Municipal Education Commission-Plateau Disciplinary Program for Medical Technology of Shanghai University of Medicine and Health Sciences (SUMHS) (grant no. 2018-2020) and the Special Program for Collaborative Innovation of SUMHS (grant no. SPCI-17-17-001).

\section{Availability of data and materials}

The datasets used and/or analyzed during the current study are available from the corresponding author on reasonable request.

\section{Authors' contributions}

YJ and JS conceived and designed the study. JW performed the experiments, analyzed the data and wrote the manuscript. ZD conducted research and prepared critical reagents. YZ performed the experiments and edited the manuscript. XZ participated in the work of animal experiment, collected and analyzed the data. All the authors have read and approved the final manuscript.

\section{Ethics approval and consent to participate}

The animal procedures were approved by the Animal Care and Use Committee of Shanghai University of Medicine and Health Sciences (Shanghai, China; permit no. 210105197306201429-kjc) and were in accordance with the Guide for the Care and Use of Laboratory Animals published by the US National Institutes of Health (NIH) (34).

\section{Patient consent for publication}

Not applicable.

\section{Competing interests}

All authors declare that they have no competing interests.

\section{References}

1. GBD 2013 Mortality and Causes of Death Collaborators: Global, regional, and national age-sex specific all-cause and cause-specific mortality for 240 causes of death, 1990-2013: A systematic analysis for the global burden of disease study 2013 . Lancet 385: 117-171, 2015.

2. GBD 2017 Causes of Death Collaborators: Global, regional, and national age-sex-specific mortality for 282 causes of death in 195 countries and territories, 1980-2017: A systematic analysis for the global burden of disease study 2017. Lancet 392: 1736-1788, 2018.

3. Braunwald E: Heart failure. JACC Heart Fail 1: 1-20, 2013.

4. Shimizu I and Minamino T: Physiological and pathological cardiac hypertrophy. J Mol Cell Cardiol 97: 245-262, 2016.

5. Zhang QJ, Tran TAT, Wang M, Ranek MJ, Kokkonen-Simon KM, Gao J, Luo X, Tan W, Kyrychenko V, Liao L, et al: Histone lysine dimethyl-demethylase KDM3A controls pathological cardiac hypertrophy and fibrosis. Nat Commun 9: 5230, 2018.

6. Savic-Radojevic A, Pljesa-Ercegovac M, Matic M, Simic D, Radovanovic S and Simic T: Novel biomarkers of heart failure. Adv Clin Chem 79: 93-152, 2017.

7. Zhang S, Yin Z, Dai FF, Wang H, Zhou MJ, Yang MH, Zhang SF, Fu ZF, Mei YW, Zang MX and Xue L: miR-29a attenuates cardiac hypertrophy through inhibition of PPARS expression. J Cell Physiol 234: 13252-13262, 2019.

8. Salisbury SA, Forrest HS, Cruse WB and Kennard O: A novel coenzyme from bacterial primary alcohol dehydrogenases. Nature 280: 843-844, 1979

9. Lu J, Chen S, Shen M, He Q, Zhang Y, Shi Y, Ding F and Zhang Q: Mitochondrial regulation by pyrroloquinoline quinone prevents rotenone-induced neurotoxicity in Parkinson's disease models. Neurosci Lett 687: 104-110, 2018. 
10. Steinberg FM, Gershwin ME and Rucker RB: Dietary pyrroloquinoline quinone: Growth and immune response in BALB/c mice. J Nutr 124: 744-753, 1994

11. Jonscher KR, Stewart MS, Alfonso-Garcia A, DeFelice BC, Wang XX, Luo Y, Levi M, Heerwagen MJ, Janssen RC, de la Houssaye BA, et al: Early PQQ supplementation has persistent long-term protective effects on developmental programming of hepatic lipotoxicity and inflammation in obese mice. FASEB J 31: 1434-1448, 2017

12. Liu Z, Sun C, Tao R, Xu X, Xu L, Cheng H, Wang Y and Zhang D: Pyrroloquinoline quinone decelerates rheumatoid arthritis progression by inhibiting inflammatory responses and joint destruction via modulating NF- $\mathrm{KB}$ and MAPK pathways. Inflammation 39: 248-256, 2016.

13. Wu R, Pan J, Shen M and Xing C: Apoptotic effect of pyrroloquinoline quinone on chondrosarcoma cells through activation of the mitochondrial caspase-dependent and caspase-independent pathways. Oncol Rep 40: 1614-1620, 2018.

14. Misra HS, Rajpurohit YS and Khairnar NP: Pyrroloquinolinequinone and its versatile roles in biological processes. J Biosci 37: 313-325, 2012.

15. Zhu BQ, Simonis U, Cecchini G, Zhou HZ, Li L, Teerlink JR and Karliner JS: Comparison of pyrroloquinoline quinone and/or metoprolol on myocardial infarct size and mitochondrial damage in a rat model of ischemia/reperfusion injury. J Cardiovasc Pharmacol Ther 11: 119-128, 2006.

16. Stites T, Storms D, Bauerly K, Mah J, Harris C, Fascetti A, Rogers Q, Tchaparian E, Satre M and Rucker RB: Pyrroloquinoline quinone modulates mitochondrial quantity and function in mice. J Nutr 136: 390-396, 2006.

17. Zhu B, Zhou H, Teerlink JR and Karliner JS: Pyrroloquinoline quinone (PQQ) decreases myocardial infarct size and improves cardiac function in rat models of ischemia and ischemia/reperfusion. Cardiovasc Drugs Ther 18: 421-431, 2004.

18. Bauerly K, Harris C, Chowanadisai W, Graham J, Havel PJ, Tchaparian E, Satre M, Karliner JS and Rucker RB: Altering pyrroloquinoline quinone nutritional status modulates mitochondrial, lipid, and energy metabolism in rats. PLoS One 6: e21779, 2011.

19. Nehra S, Bhardwaj V, Bansal A, Chattopadhyay P and Saraswat D: Nanocurcumin-pyrroloquinoline formulation prevents hypertrophy-induced pathological damage by relieving mitochondrial stress in cardiomyocytes under hypoxic conditions. Exp Mol Med 49: e404, 2017

20. Nehra S, Bhardwaj V, Bansal A and Saraswat D: Combinatorial therapy of exercise-preconditioning and nanocurcumin formulation supplementation improves cardiac adaptation under hypobaric hypoxia. J Basic Clin Physiol Pharmacol 28: 443-453, 2017.

21. Xu F, Yu H, Liu J and Cheng L: Pyrroloquinoline quinone inhibits oxygen/glucose deprivation-induced apoptosis by activating the PI3K/AKT pathway in cardiomyocytes. Mol Cell Biochem 386: 107-115, 2014.

22. Gong W, Duan Q, Cai Z, Chen C, Ni L, Yan M, Wang X, Cianflone K and Wang DW: Chronic inhibition of cGMP-specific phosphodiesterase 5 suppresses endoplasmic reticulum stress in heart failure. Br J Pharmacol 170: 1396-1409, 2013.

23. Facundo HDTF, Brainard RE, Caldas FRL and Lucas AMB: Mitochondria and cardiac hypertrophy. Adv Exp Med Biol 982: 203-226, 2017.

24. Lyon RC,ZanellaF,Omens JHand SheikhF:Mechanotransduction in cardiac hypertrophy and failure. Circ Res 116: 1462-1476, 2015.

25. Garcia-Redondo AB, Aguado A, Briones AM and Salaices M: NADPH oxidases and vascular remodeling in cardiovascular diseases. Pharmacol Res 114: 110-120, 2016.

26. Chen F, Wang H, Zhao J, Yan J, Meng H, Zhan H, Chen L and Yuan L: Grape seed proanthocyanidin inhibits monocrotaline-induced pulmonary arterial hypertension via attenuating inflammation: In vivo and in vitro studies. J Nutr Biochem 67: 72-77, 2019.

27. Han Q, Liu Q, Zhang H, Lu M, Wang H, Tang F and Zhang Y: Simvastatin improves cardiac hypertrophy in diabetic rats by attenuation of oxidative stress and inflammation induced by calpain-1-mediated activation of nuclear factor- $\kappa \mathrm{B}(\mathrm{NF}-\kappa \mathrm{B})$. Med Sci Monit 25: 1232-1241, 2019.

28. Shi R, Wei Z, Zhu D, Fu N, Wang C, Yin S, Liang Y, Xing J, Wang $X$ and Wang $Y$ : Baicalein attenuates monocrotaline-induced pulmonary arterial hypertension by inhibiting vascular remodeling in rats. Pulm Pharmacol Ther 48: 124-135, 2018 .
29. Zhang C, Wang F, Zhang Y, Kang Y, Wang H, Si M, Su L, Xin X, Xue F, Hao F, et al: Celecoxib prevents pressure overload-induced cardiac hypertrophy and dysfunction by inhibiting inflammation, apoptosis and oxidative stress. J Cell Mol Med 20: 116-127, 2016.

30. Li Y, Xia J, Jiang N, Xian Y, Ju H, Wei Y and Zhang X: Corin protects $\mathrm{H}_{2} \mathrm{O}_{2}$-induced apoptosis through PI3K/AKT and NF- $\mathrm{KB}$ pathway in cardiomyocytes. Biomed Pharmacother 97: 594-599, 2018.

31. Guo Z, Lu J, Li J, Wang P, Li Z, Zhong Y, Guo K, Wang J, Ye J and Liu P: JMJD3 inhibition protects against isoproterenol-induced cardiac hypertrophy by suppressing $\beta$-MHC expression. Mol Cell Endocrinol 477: 1-14, 2018.

32. Sasaki CY,Barberi TJ, Ghosh P and Longo DL: Phosphorylation of RelA/p65 on serine 536 defines an I $\{$ kappa\} $B$ \{alpha\}-independent NF-\{kappa\}B pathway. J Biol Chem 280: 34538-34547, 2005.

33. Davidson M, Nesti C, Palenzuela L, Walker WF, Hernandez E, Protas L, Hirano M and Isaac ND: Novel cell lines derived from adult human ventricular cardiomyocytes. J Mol Cell Cardiol 39: 133-147, 2005.

34. National Institutes of Health: Guide for the care and use of laboratory animals. 8th eidition. National research council (US) committee for the update of the guide for the care and use of laboratory animals. Washington (DC), National Academies Press (US), 2011

35. Yoshida T, Yamashita M, Horimai $C$ and Hayashi M: Kruppel-like factor 4 protein regulates isoproterenol-induced cardiac hypertrophy by modulating myocardin expression and activity. J Biol Chem 289: 26107-26118, 2014

36. Li C, Huang D, Tang J, Chen M, Lu Q, Li H, Zhang M, Xu B and Mao J: ClC-3 chloride channel is involved in isoprenaline-induced cardiac hypertrophy. Gene 642: 335-342, 2018.

37. Ren J, Zhang N, Liao H, Chen S, Xu L, Li J, Yang Z, Deng W and Tang Q: Caffeic acid phenethyl ester attenuates pathological cardiac hypertrophy by regulation of MEK/ERK signaling pathway in vivo and vitro. Life Sci 181: 53-61, 2017.

38. Sato H, Suzuki JI, Aoyama N, Watanabe R, Kaneko M, Shiheido Y, Yoshida A, Wakayama K, Kumagai H, Ikeda Y, et al: A periodontal pathogen porphyromonas gingivalis deteriorates isoproterenol-induced myocardial remodeling in mice. Hypertens Res 40: 35-40, 2017.

39. Lucas AMB, de Lacerda Alexandre JV, Araújo MTS, David CEB, Viana YIP, Coelho BN, Caldas FRL, Varela ALN, Kowaltowski AJ and Facundo HT: Diazoxide modulates cardiac hypertrophy by targeting $\mathrm{H} 2 \mathrm{O} 2$ generation and mitochondrial superoxide dismutase activity. Curr Mol Pharmacol: Jul 23, 2019 (Epub ahead of print).

40. Gong D, Geng C, Jiang L, Aoki Y, Nakano M and Zhong L: Effect of pyrroloquinoline quinone on neuropathic pain following chronic constriction injury of the sciatic nerve in rats. Eur J Pharmacol 697: 53-58, 2012.

41. Xiao Y, Yang Z, Wu QQ, Jiang XH, Yuan Y, Chang W, Bian ZY, Zhu JX and Tang QZ: Cucurbitacin B protects against pressure overload induced cardiac hypertrophy. J Cell Biochem 118: 3899-3910, 2017.

42. Livak KJ and Schmittgen TD: Analysis of relative gene expression data using real-time quantitative PCR and the 2(-Delta Delta C(T)) method. Methods 25: 402-408, 2001.

43. Kim B: Western blot techniques. Methods Mol Biol 1606: 133-139, 2017.

44. Elefantova K, Lakatos B, Kubickova J, Sulova Z and Breier A: Detection of the mitochondrial membrane potential by the cationic dye JC-1 in L1210 cells with massive overexpression of the plasma membrane ABCB1 drug transporter. Int J Mol Sci 19: pii: E1985, 2018.

45. Li D, Ye Y, Lin S, Deng L, Fan X, Zhang Y, Deng X, Li Y, Yan H and Ma Y: Evaluation of deoxynivalenol-induced toxic effects on DF-1 cells in vitro: Cell-cycle arrest, oxidative stress, and apoptosis. Environ Toxicol Pharmacol 37: 141-149, 2014.

46. Xia W, Zhuang L and Hou M: Role of lincRNA-p21 in the protective effect of macrophage inhibition factor against hypoxia/serum deprivation-induced apoptosis in mesenchymal stem cells. Int J Mol Med 42: 2175-2184, 2018.

47. Althurwi HN, Tse MM, Abdelhamid G, Zordoky BN, Hammock BD and El-Kadi AO: Soluble epoxide hydrolase inhibitor, TUPS, protects against isoprenaline-induced cardiac hypertrophy. Br J Pharmacol 168: 1794-1807, 2013.

48. Rothschild DE, McDaniel DK, Ringel-Scaia VM and Allen IC: Modulating inflammation through the negative regulation of NF- $\kappa$ B signaling. J Leukoc Biol, Feb 1, 2018 (Epub ahead of print). 
49. Zeng MY, Miralda I, Armstrong CL, Uriarte SM and Bagaitkar J: The roles of NADPH oxidase in modulating neutrophil effector responses. Mol Oral Microbiol 34: 27-38, 2019.

50. Singh AK, Pandey SK and Naresh Kumar G: Pyrroloquinoline quinone-secreting probiotic Escherichia coli Nissle 1917 ameliorates ethanol-induced oxidative damage and hyperlipidemia in rats. Alcohol Clin Exp Res 38: 2127-2137, 2014.

51. Lu H, Shen J, Song X, Ge J, Cai R, Dai A and Jiang Z: Protective effect of pyrroloquinoline quinone (PQQ) in rat model of intracerebral hemorrhage. Cell Mol Neurobiol 35: 921-930, 2015.

52. Zhou XQ, Yao ZW, Peng Y, Mao SS, Xu D, Qin XF and Zhang RJ: PQQ ameliorates D-galactose induced cognitive impairments by reducing glutamate neurotoxicity via the GSK-3ß/Akt signaling pathway in mouse. Sci Rep 8: 8894, 2018.

53. Mitchell P: Coupling of phosphorylation to electron and hydrogen transfer by a chemi-osmotic type of mechanism. Nature 191: 144-148, 1961

54. Chen LB: Mitochondrial membrane potential in living cells. Annu Rev Cell Biol 4: 155-181, 1988.

55. Sakamuru S, Attene-Ramos MS and Xia M: Mitochondrial membrane potential assay. Methods Mol Biol 1473: 17-22, 2016.

56. Mohamed TMA, Ang YS, Radzinsky E, Zhou P, Huang Y, Elfenbein A, Foley A, Magnitsky S and Srivastava D: Regulation of cell cycle to stimulate adult cardiomyocyte proliferation and cardiac regeneration. Cell 173: 104-116.e12, 2018.

57. Foglia MJ and Poss KD: Building and re-building the heart by cardiomyocyte proliferation. Development 143: 729-740, 2016.

58. Kasahara $\mathrm{T}$ and Kato T: Nutritional biochemistry: A new redox-cofactor vitamin for mammals. Nature 422: 832, 2003.

59. Bishop A, Gallop PM and Karnovsky ML: Pyrroloquinoline quinone: A novel vitamin? Nutr Rev 56: 287-293, 1998.

60. Yang C, Yu L, Kong L, Ma R, Zhang J, Zhu Q, Zhu J and Hao D: Pyrroloquinoline quinone (PQQ) inhibits lipopolysaccharide induced inflammation in part via downregulated NF- $\kappa \mathrm{B}$ and p38/JNK activation in microglial and attenuates microglia activation in lipopolysaccharide treatment mice. PLoS One 9: e109502, 2014.
61. Hong HQ, Lu J, Fang XL, Zhang YH, Cai Y, Yuan J, Liu PQ and Ye JT: G3BP2 is involved in isoproterenol-induced cardiac hypertrophy through activating the NF- $\mathrm{NB}$ signaling pathway. Acta Pharmacol Sin 39: 184-194, 2018.

62. Song SB, Jang SY, Kang HT, Wei B, Jeoun UW, Yoon GS and Hwang ES: Modulation of mitochondrial membrane potential and ROS generation by nicotinamide in a manner independent of SIRT1 and mitophagy. Mol Cells 40: 503-514, 2017.

63. Ortiz-Avila O, Esquivel-Martinez M, Olmos-Orizaba BE, Saavedra-Molina A, Rodriguez-Orozco AR and Cortés-Rojo C: Avocado oil improves mitochondrial function and decreases oxidative stress in brain of diabetic rats. J Diabetes Res 2015: 485759, 2015.

64. Chikando A, Boyman L, Khairallah R, Williams GSB, Kettlewell S, Ward CW, Smith G, Kao J and Lderer WJ: ROS and mitochondrial membrane potential dependent modulation of calcium signaling in the heart. Biophys J 104: 361, 2013.

65. Yan B, Sun Y and Wang J: Depletion of ubi A prenyltransferase domain containing 1 expression promotes angiotensin II-induced hypertrophic response in AC16 human myocardial cells via modulating the expression levels of coenzyme Q10 and endothelial nitric oxide synthase. Mol Med Rep 16: 6910-6915, 2017.

66. Zhang W, Zhang Y, Ding K, Zhang H, Zhao Q, Liu Z and Xu Y: Involvement of JNK1/2-NF- $\mathrm{kBp} 65$ in the regulation of HMGB2 in myocardial ischemia/reperfusion-induced apoptosis in human AC16 cardiomyocytes. Biomed Pharmacother 106: 1063-1071, 2018.

This work is licensed under a Creative Commons Attribution-NonCommercial-NoDerivatives 4.0 International (CC BY-NC-ND 4.0) License. 